\section{A glimpse inside the Sun}

\section{Sunquakes: Probing the Interior}

of the Sun

by Jack B. Zirker

Johns Hopkins University Press: 2003.

288 pp. \$29.95, £22

\section{Jørgen Christensen-Dalsgaard}

"What appliance can pierce through the outer layers of a star and test conditions within?" asked Arthur Eddington in his book The Internal Constitution of the Stars (Cambridge University Press, 1926). In the case of the Sun, this question has been answered dramatically in the past few decades by the emergence of helioseismology — and there are high hopes that such investigations could be extended to other stars.

The surface of the Sun shows a variety of dynamic phenomena, largely associated with convection, where hot plasma rises to the surface. These dominate energy transport in the convection zone, which occupies the outer part of the Sun. It is the convective cells that give the Sun's surface its granular appearance. These and other motions are also reflected in the velocity of the solar atmosphere, as measured through the Doppler effect.

Variations in the Doppler velocity at the solar surface may have been noted in 1916 by Harry Plaskett, and were unambiguously detected by A. B. Hart in about 1955. In 1961, Robert Leighton, John Evans and colleagues revealed that this signal seemed to be oscillating with a period of about five minutes. The physical nature of these oscillations remained uncertain for more than a decade, until in 1970 Roger Ulrich, and independently John Leibacher and Robert Stein, proposed that the oscillations arise from trapped acoustic modes resonating in the solar interior. This was confirmed by Franz-Ludwig Deubner in 1975 and, a little later, by Edward Rhodes and his collaborators. With these results, and the detection in the late 1970s of fiveminute oscillations propagating throughout the entire Sun, the potential for using observations of these surface oscillations to probe the solar interior was evident, and helioseismology was born.

In his rich and enjoyable book, Jack Zirker presents the history of helioseismology, from the work of Leighton and Evans to current detailed investigations of the Sun's internal structure and dynamics. His discussion of the early days quite rightly includes the apparent detection of oscillations in the

solar diameter by Henry Hill and his group, and the observation of an enigmatic 160minute oscillation. Although these results were not confirmed by later investigations, they did help to inspire the subsequent developments. Descriptions of the investigations of flows in the solar convection zone associated with the solar magnetic cycle, and the peculiar periodic variations in rotation near the base of the convection zone, bring the book essentially up to date. Also covered is the development of asteroseismology, including the impressive results obtained from analysis of oscillations of white dwarfs, and the early results from emerging studies of solar-like oscillations in other stars.

A crucial feature of helioseismology is the way in which it can throw light on other aspects of the solar interior and the physical processes that control it. A key problem in astrophysics since 1968 has been the discrep-$$
\text { (a) }
$$
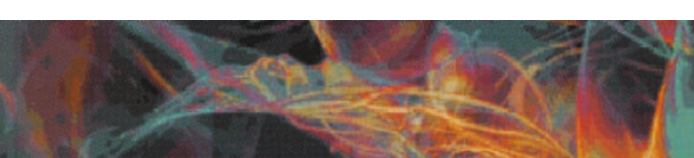

of solar magnetic activity. As discussed by Zirker, helioseismic determinations of solar internal rotation were not consistent with initial numerical models that had attempted to describe the dynamics of the solar convection zone and the origin of the variation of the solar surface rotation rate with latitude on the Sun. This led to a revision of the models of the generation of the magnetic field, which now seems likely to be dominated by the region of strong radial variation in the rotation rate at the base of the convection zone. Also, more sophisticated models of the interaction between convection and rotation, which better capture the turbulent nature of the flow, are beginning to yield results in accordance with the helioseismic inferences. And finally, local helioseismology has provided information about the subsurface structure of solar active regions and their emergence, and even led to monitoring of solar activity on the far side of the sun.

Zirker's excellent book combines the development of helioand asteroseismology with a lucid and detailed overview of modern solar physics. The text is at a level that is accessible to the interested layperson, with more technical aspects discussed in a substantial set of notes. There are a few minor inconsistencies and misprints, typically in names, and it would have been useful to have included a brief bibliography for those readers interested in delving further into particular topics.

The book is wholeheartedly recommended. Older partici-

ancy between the predicted rate of production of neutrinos in the solar core and the rate of neutrino detections in several independent experiments. This initially raised suspicions about possible problems in solar modelling, and various changes to the models were proposed to bring the neutrino predictions into line with the observations. However, even early helioseismic results strongly indicated that these modified models could not be right, and that therefore some other explanation was needed for the measured neutrino flux. Zirker gives a very clear presentation of this problem and its final solution: the Sudbury Neutrino Observatory in Canada recently demonstrated that the so-called electron neutrinos generated by nuclear reactions in the solar core undergo partial transformation to other neutrino types en route to the Earth, the combined neutrino flux being in full agreement with the models.

Rotation and other flows in the solar interior are closely related to the generation of the solar magnetic field and the 11-year cycle in the number of sunspots and other pants in the field will enjoy being reminded of how helioseismology has developed; younger scientists get an introduction to the history of their subject, and the general reader is given a fascinating overview of one of the newest and most active fields of astrophysics, including enjoyable cameos of some of the participants.

The appearance of this book is also timely; helioseismology is a well-consolidated field and asteroseismology is poised on the brink of full emergence. It is unfortunate that the European Space Agency's Eddington mission, which was to have carried out asteroseismic investigations for a large number of stars and searched for extra-solar planets, has been cancelled. Should it be reinstated, we would obtain a fuller answer to Eddington's original question, and our understanding of the interior properties of other stars and their place in the Universe would be greatly advanced.

Jørgen Christensen-Dalsgaard is at the Theoretical Astrophysics Center, Danish National Research Foundation, and the Institute of Physics and Astronomy, University of Aarhus, DK 8000 Aarhus C, Denmark. 